and the child was restless, refusing all nourishment when first offered. About four hours after the operation the child was fed from the breast and subsequently on three occasions. About 14 hours after the operation the bowels were moved fairly well, a natural motion was passed, but the child failed rapidly about 24 hours after laparotomy and died from shock six hours later. Post mortem the peritoneum appeared to be quite normal, there was no recurrence of intussusception, and some adhesions had formed between the mesoappendix and the mesentery. There can be little doubt that earlier operation should bave saved this patient.

Remarks by Mr. RAY. - The surgical treatment of acute intussusception presents many features of great interest and although the results of treatment by laparotomy and reduction, where this is possible, are not always so good as might be expected, failure is usually due to the fact that operation has been postponed until the involved portion of gut has been most seriously damaged. or that the initial shock and peritonism have caused too deep an impression to be removed by any treatment In THE LANCET of Nov. 12th, 1898 (p. 1262), I reported a case of acute ileo-cæcal intussusception where laparotomy and reduction revealed a serious lesion in the ascending colon, and where death followed from continued shock in nine hours after operation. Since reporting that case the above cases of acute intussusception have been under my care, all being of the ileo-cæcal type. In three instances laparotomy and reduction were carried out and resulted in two recoveries and one death. In the remaining case the intussusception was irre ducible, and, moreover, the ascending colon forming the intussuscipiens bad perforated in two places; here the entire intussusception was excised and end-to-end anastomosis was performed by the use of Murphy's button, but this case terminated fatally. The duration of intussusception was 12 hours and two days respectively in the two successful cases, whilst it was two and a half days and five days respectively in the two fatal cases. The irreducible variety occurred in a child, aged seven weeks; the remaining children were aged eight months, seven months, and five months respectively and in each of these the mother fed the child from the breast after operation. In all four cases either a more or less definite tumour or the signe de Dance (loss of resistance to palpation over the right iliac fossa) conld be made out and in all the cases an acute onset was speedily followed by the passage of blood and mucus per anum. It will be noticed that in three cases the patients were males and in one case the patient was a female. In the accompanying table will be found the leading features of each case.

\section{STATE HOSPITAL, JAMMU.}

\section{TWO CASES OF RENAL SURGERY.}

(Under the care of Lieutenant.Colonel JoshuA DukE, I.M.S., Resident Surgeon in Kashmir.)

THE difficulty of detection of a renal calculus is of ten very great and needling has in many cases utterly failed to discover the presence of a stone which has been found later. The Roentgen rays are sometimes of value, especially when the stone is composed of calcium oxalate, for a uric acid stone gives but a very faint shadow. Probably the best procedure to adopt when palpation of the kidney gives no indication of the presence of a stone is to make an incision along the convexity of the kidney, laying open the cavity of its pelvis. In this way the whole of the organ can be very thoroughly explored and the stone can almost certainly be found.

CASE 1. Nephrolithotomy.-A sepoy in the Imperial Service troops, Kashmir, came before Lieutenant-Colonel Doke at an annual invaliding board in December, 1898. The patient then complained of severe pain in the right hypochondriac and lumbar regions. He said that this pain rendered his life a burden to him and that he could serve no longer as a soldier. Inquiry proved that he had previously undergone an operation at Gilgit for the relief of the pain and he showed a scar in the right loin. He was therefore admitted to hospital for further examination and operative treatment. A telegram was sent to the agency surgeon at Gilgit (400 miles distant) inquiring whether the man's statement was correct and a reply was received by wire saying that an operation bad bren performed-the kidney had been manipulated but no stone was found. Owing to the kindness of Major Roberts, I.M.S., LieutenantColonel Duke is able to give the patient's previous history and a note on the primary operation.

The patient, who was 28 years of age, was admitted to the Military Hospital, Gilgit, on Jan. 23rd, 1899. He complained of pain in the right loin. Sometimes this pain was situated in the loin and at other times over the sacro-iliac joint, radiating to the other side. The urine contained albomin. As the case progressed the amount of albumin varied from one-eighth to one-sixth, sometimes almost disappearing. There was no blood. About the middle of April he began to have pain radiating from the loin into the right groin and testicle. As there was no improvement under treatment it was decided to explore the kidney on the supposition of stone, although the amount of albumin in the urine was not in favour of this.

An operation was performed on May 8th, 1899. The usual oblique incision was made in the right loin and the peritoneal fat was exposed. This was stripped off and the anterior surface and the hilum were fully exposed to the touch of the finger. The kidney was judged to be small and no prominence could be made out on any part. The posterior surface and ureter were also explored. It was not judged necessary to try the needle. Deep catgut sutures were placed in the muscles and a drainage-tube was inserted in the wound which healed without any complication. After the operation there was an interval withont pain in the loin, but this symptom returned. The patient was sent back to Kashmir and Jammu, leaving Gilgit on June 16th, 1899.

On August 24th, at Jammu, the mine was of specific gravity 1026 and contained one-quarter albumin and lithates in abundance. He was re-admitted into the State Hospitil, Jammu, on Dec. 26th, 1899. There was then severe and almost constant pain in the right hypochonoriac region and in the right loin. The pain was aggravated by pressure before and behind. The urine was normal in every respect.

The second operation was performed on Jan. 1st, 1900 . The right loin was opened for the second time. The incision was now made parallel with, but fully balf an inch posterior to, the primary scar. The renal fat was exposed and some was torn out, and the kidney was brought well into view. Careful palpation revealed nothing. Needling was then commenced and six punctures were deeply made in various positions. Nothing could be felt and Lientenant-Colonel Duke began to despair of finding anything. At the seventh puncture, however, made from below upwards a stone was felt and then struck on audibly. A deep incision was made and a Thompson's sound was passed. This at first entered the ureter, running easily; it was then withdrawn and turned and struck the stone in the upper calyx of the kidrey with a metallic noise. The opening was then enlarged, free bleed. ing ensuing. The finger was now passed into the hilum on to the stone. The calculus, however, was firmly adherent and its pointed apex was imbedded, so that it had to be slowly peeled off and loosened. It was then removed with forceps. The hæmorrhage was controlled by sponge-pressure and after it had ceased a drainage-tube was inserted and the wound was firmly closed. The stone, which was roughly triangular in shape, weighed on removal 36 grains. I he patient did well after the operation, though a good deal of blood was passed in the urine, a symptom which continued until the ninth day. On Jan. 15th the drainage-tube, which had been frequently shortened, was taken out and the sutures were removed. There was slight sweet discharge. The urine contained mucus and lithates but no blood or pns. Un Feb. 1st the wound was soundly healed and the patient was up and about.

CaSE 2. Nephrotomy. - A Hindu male, aged 35 years, was admitted into the State Hospital, Jammu, on March $16 \mathrm{rh}$, 1900. Ten years ago be had an acute attack of pain in the left lumbar region which lasted for 12 hours and was relieved by taking a warm drink, prescribed by a native physician. The pain was associated with a sensation of something rough passing down from the kidney and the patient thought that his left testicle was drawn up. Three years later he passed some sand and gravel with his urine. Four years subsequently he had another attack of lumbar pain similar to the first. There was no history of hæmaturia or definite pain with drawing up of the testicle. He had never had retention of the urine. His general health had failed since these attacks. On admission there was marked tenderness on pressure over the lower border of the right kidney below the rib, the patient wincing when the thumb was pressed on. 
The pain was increased when the hand was placed over the abdomen and pressure was made from behind. He com. plained of a sensation of something rough moving in the region of the kidney. The above were the only symptoms. The urine was of specific gravity 1029 and was healthy in every way.

An operation was performed on March 21st, 1900. The kidney was exposed in the left lumbar region by an incision four inches in length. About two inches of perirenal fat were removed and the kidney was brought into view, but not ontside the loin. On drawing the lower end of the kidney into the wound a small cyst of the size of a pea was seen. This was needled and a little yellow serum was evacuated. On passing the needle in deeper some more of the same serum (almost pus) oozed up. The needle was then passed in deeply at the lower fourth and struck on what felt exactly like a stone or concretion. A cataract knife was passed down over the needle, which was withdrawn, but no stone was felt, although the needle had given the actnal sensation of hitting a stone. A free incision was then made in the lower part of the organ and the finger was passed in but nothing could be felt. A sound was then inserted which, however, did not pass down the nreter owing probably to the incision being in the lower third of the organ. The finger was then thrust on the hilum. All the calyces were explored but no stone could be felt with the finger inside and careful palpation outside. Several small nodules or lumps were felt; all these were needled. They were nrobably small cysts of the same nature as that seen on the surface. The operation had therefore unwillingly to be abandoned, although the operator conld have sworn that a stone or concretion had been originally struck on. It may be noted that while exploring with the finger inside the respiration twice stopped. The hæmorrhage in this case was very trifling. A drainage tube having been inserted, the deeper structures were drawn togetber by catgut sutures and the wound was firmly closed by six silk sutures. One-third of a grain of morphia was injected after the operation, as the kidney had been severely handled.

On March 22nd the patient passed a restless night. The dressings, which were soaked with bloody serum, were changed. The temperature was $102^{\circ} \mathrm{F}$. The patient had a cough and there was viscid white expectoration pointing to pneumonia. On the 23rd the temperature was normal, the pain was much less, and the general condition was satisfactory. On the 24th he was doing well. On the 27th the urine was still smoky. There was a discharge from the wound with a uriniferous smell. The cough was still present, with frothy expectoration; the respiratory sounds were clear over both lungs. On April 3rd and 4th there was a rise of temperature in the evening. On the 5 th the dressings were still soaked with a uriniferous discharge. The lower two-thirds of the wound had healed but there was no attempt at union in the upper third, which was a fistulous track four inches deep. The urine was now quite clear. There was an erysipelatous blush around the wound and the interscapular region. The patient was removed to a separate room, the ward in which he had been treated being rather overcrowded and occupied by some very foul cases, including faminestricken patients with sloughing ulcers. To continue briefly, the patient was brought to death's door by this and subsequent erysipelatous attacks from which recovery was slow, and he was not discharyed from the hospital until June 3rd. The wound had then completely healed, the process of union being retarded by consecutive attacks of erysipelas. There had been no recurrence of pain in the loin ever since the operation and the deep-seated tenderness over the kidney was no longer complained of.

Remarks by Lientenant-Colonel DukE.-Considering the enormous preponderance of cases of stone in the bladder in India and the extensive removal of calculi by litholapaxy, it is curious that stone in the kidney is comparatively rarely met with, and surgeons in India do not appear to have the opportunities of exploring the kidney that fall to their confrères at home. When Mr. Henry Morris, the great pioneer and exponent of kidney surgery in England, was house surgeon at Guy's Hospital I had the bonour of being dresser at that institution; but during my period of studentship I cannot recall seeing a kidney cut down on, nor, unfortunately, have I ever seen this operation performed by a skilled surgeon. In Indian civil and hospital practice, however, one has to take every case that comes to hand. As bearing on this subject since my case in 1895 of nephrotomy for neuralgia of the kidney, ${ }^{1}$ though I have performed litholapaxy many times the above two cases are the only ones that have occurred in my practice. The points of interest in Case 1 are (1) the presence of a large though transient quantity of albumin, and (2) the fact that simple manipulation failed to discover the presence of a calculus. In Case 2 the operation only revealed cystic disease of the kidney. No stone was found although the kidney was most freely handled and examined. It is just possible that a small stone was pushed on or slipped into the ureter, which was not carefully catheterised.

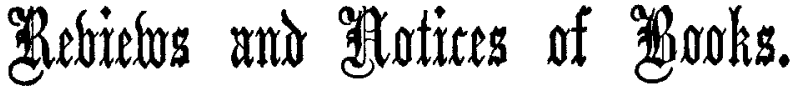

Manual of Midwifery. By W. E. FotherGILL, M.A., M.D. Edin. Second edition. With donble Coloured Plate and 76 Illustrations in the text. Edinburgh: W. F. Clay. 1900. Pp. 506. 8vo. Price 9s. net.

WE are glad to see by the appearance of a second edition that this very excellent little manual has met with some success. Owing to the short time that has elapsed since its first appearance very little alteration has been required to bring it up to date. The author has succeeded in doing this without materially adding to its size, the increase in the number of pages being only about 20 . Amongst the additions we notice a short account of Van Tussenbroek's case of ovarian pregnancy. The section devoted to the subject of posture during labour has been largely added to and diagrams have been introduced to show the advantages derived from an adoption of Walcher's position. $D$ r. Fotbergill arrives at the following conclusions with regard to the position of the patient: 1. For labour presenting no difficulty the left lateral posture is best. 2. If the head be arrested at the brim Walcher's posture, legs hanging down. 3 . Head arrested at the outlet of the long pelvis, lithotom posture, legs pressed against the abdomen. 4. Head arrested on the perineum, legs extended at the hips.

In a short account of the organisms most commonly met with in cases of puerperal infection too much is, perhaps, said about the bacillus coli communis. It has yet to be proved with certainty that either the staphylococcus or the bacillus coli communis can give rise to septic infection as opposed to septic intoxication. With the statement that most cases of "fever during the puerporium are not cases of puerperal septicæmia" but are due to the absorption of poisonous substances from the alimentary canal we entirely agree.

In an appendix Dr. Fothergill gives a short account of the so-called hepatic toxæmia of pregnancy. Its relation to the minor and major disorders of pregnancy and its proper treatment are fully discussed.

Several new figures have been added, including the microscopic appearances of the decidua and villi and illustrations of fleshy moles. We would like to suggest to the author that in place of Fig. 9-an inaccurate diagram of the placenta-he should insert in another edition either a new and accurate diagram or one of the very excellent drawings of the placenta to be found in Varnier's "Obstétrique Journalière." The book contains a clear and good account of the facts of midwifery and is well worth reading.

Ersentials of Diagnosis. By Solomon Solis-CoHen, M.D.r. and Augustus A. Eshner, M.D. Second edition, revised and enlarged. Illustrated. London: Henry Kimpton. 1900. Pp. 391.

THE first edition of this little work, published in America in 1892, constituted one of the series known as Saunders's Question-compends. Its popularity among medical students. in America to-day justifies a second edition and warrants its inclusion in Kimpton's Students' Series. In its present form

1 Vide The LavceT, August 17th, 1895, p. 407. 